\title{
Association between IL17A/IL17RA Gene Polymorphisms and Susceptibility to Alopecia Areata in the Korean Population
}

\author{
Bark-Lynn Lew, M.D., Hee-Ryung Cho, M.D., Sik Haw, M.D., Hwi-Jun Kim, M.D., \\ Joo-Ho Chung, M.D. ${ }^{1}$, Woo-Young Sim, M.D. \\ Departments of Dermatology and ${ }^{1}$ Pharmacology, Kyung Hee University College of Medicine, Seoul, Korea
}

\begin{abstract}
Background: Alopecia areata is marked by autoimmune assault on the hair follicle resulting in hair loss. T helper 17 cell subset has important roles in protecting the host against extracellular pathogens, however, also promotes inflammatory pathology in autoimmune disease, and it expresses both interleukin (IL)-17A and IL-17F, which can signal via the IL-17 receptor A. Objective: To investigate the significance of IL17A and IL17RA gene polymorphisms in the susceptibility to alopecia areata. Methods: We conducted case-control association study of 238 alopecia areata patients and 270 matched healthy controls. Allele frequency of total 2 single nucleotide polymorphims in the IL17A gene and 4 single nucleotide polymorphims in the IL17RA gene were studied. The statistical analyses were performed according to onset age, the presence of familyhistory, clinical subtypes, and presence of nail involvement or body hair involvement. Results: One single nucleotide polymorphim (rs879577) of IL17RA gene showed significant difference between alopecia areata patients group and controls group ( $p=$ 0.0288). One single nucleotide polymorphim (rs4819554) of IL17RA gene showed significant difference between the early onset and late onset alopecia areata $(p=0.0421)$. Conclusion: IL17RA gene polymorphism might contribute to
\end{abstract}

Received October 11, 2011, Revised November 28, 2011, Accepted for publication November 30, 2011

Corresponding author: Woo-Young Sim, M.D., Department of Dermatology, Kyung Hee University Hospital at Gang-dong, 149 Sangildong, Gangdong-gu, Seoul 134-727, Korea. Tel: 82-2-440-7329, Fax: 82-2-440-7336, E-mail: bellotte@hanmail.net

This is an Open Access article distributed under the terms of the Creative Commons Attribution Non-Commercial License (http:// creativecommons.org/licenses/by-nc/3.0) which permits unrestricted non-commercial use, distribution, and reproduction in any medium, provided the original work is properly cited. the increased susceptibility to alopecia areata in Korean population, and ILI7RA gene polymorphism may be associated with onset age. (Ann Dermatol 24(1) 61 65, 2012)

\section{-Keywords-}

Alopecia areata, Interleukin-17A, Interleukin-17 receptors A, Single nucleotide polymorphism

\section{INTRODUCTION}

Alopecia areata (AA) is an organ-specific autoimmune disease characterized by T-cell infiltrates and cytokine production around anagen-stage hair follicles ${ }^{1,2}$. There is evidence for loss of immune privilege coupled with $\mathrm{T}$ cell mediated attack of hair follicle autoantigens ${ }^{3}$. The role of $T$ helper (Th) 1 and Th2 cytokines in the pathogenesis of AA have not been fully established. The evidence for the role of cytokines and T-cells in AA includes the beneficial effects of systemic steroids and calcineurin inhibitors ${ }^{4,5}$. Autoimmune diseases result from many factors, including the interaction of the genetic background of the immune system with the environment.

AA being linked to genetic factors is supported by the fact that $4 \%$ to $28 \%$ of patient's have a family history ${ }^{6}$. It is probable that $\mathrm{AA}$ and other autoimmune diseases are polygenic, with multiple potential routes of genetic susceptibility $^{7}$. Many studies have been done on the linkages of various genes to AA. The most extensive work has been established on linkages with the human leukocyte antigen (HLA) hapolotypes and less extensive work has also been done on the immune response and other genes ${ }^{6}$. In addition to HLA genes, there are several known 
genetic polymorphisms in cytokine receptors and antigen-processing molecules.

Several studies in the late 1990s implicated interleukin (IL)-17 in the pathogenesis of autoimmunity. Elevated IL-17 levels were found in rheumatoid arthritis, systemic lupus erythematosus, and psoriasis patients, although it was not clear from these studies how important a role it may play in disease pathogenesis ${ }^{8,9}$. The IL-17 cytokine family consists of six members: IL-17A, IL-17B, IL-17C, IL-17D, IL-17E/IL-25, and IL-17F. The best characterized member of this family is IL-17A, and its expression facilitates the definition of a newly discovered lineage of CD4 + effector T cells, the Th17 subset $^{10-14}$. IL-17A is the founding member of a novel family of inflammatory cytokines that plays a critical role in the pathogenesis of many autoimmune diseases, including multiple sclerosis and experimental autoimmune encephalomyelitis, but it was not clear from these studies how important a role it may play in disease pathogenesis ${ }^{9}$. The receptor for IL-17, which is referred to as IL-17R from here on, is abundantly expressed by all cells of the immune system, and stimulation of various cell types with IL-17 can induce the expression of other cytokines such as IL-1 $\beta$, TNF and IL-6, and the chemokines IL-8 and macrophage inflammatory protein 1. In contrast to the restricted set of cells that produce IL-17, mRNA of the IL-17R is expressed ubiquitously in many cell types and shares no homology with any other cytokine receptor family ${ }^{15}$.

The role of IL-17 on the pathogenesis of AA has not been studied yet, but we suggest that IL-17 may be associated with AA like other autoimmune diseases. The purpose of this study is to determine whether variation in the IL17A/IL17RA gene contributes to the risk of AA in Korean population.

\section{MATERIALS AND METHODS}

\section{Study subjects}

AA patients and control subjects in Kyung Hee University Hospital at Gang-dong (Kyung Hee East West Neo Medical Center) were enrolled in this study. The control subjects were recruited after they had been determined as mentally and physically healthy in a general health checkup program. AA patients were diagnosed by clinical features and physical examination including pull test and microscopic analysis of hairs. However, diagnosis of $\mathrm{AA}$ was confirmed by skin biopsy in some cases. A careful history was taken from each patient and concerned general health, including the existence of previous $A A$, triggering factors, the presence of autoimmune disease, or atopy, and a family history of AA or autoimmune disease.
A serologic work-up including an anemia study, venereal desease research laboratory test, antinuclear antibodies, a thyroid function test, and androgenic hormones, such as testosterone, estradiol, luteinizing hormone, and folliclestimulating hormone was carried out for all patients. Two hundred and seventy healthy controls (144 males and 126 females, average age at survey: 35.7) were included. Healthy controls did not have any known diseases or symptoms.

Informed consent was obtained from each subject, and the study was approved by the Institutional Review Board of Kyung Hee University Hospital at Gang-dong. Genomic DNA was prepared from peripheral blood using a genomic DNA isolation reagent kit (Core-BioSystem, Seoul, Korea).

\section{Single nucleotide polymorphism (SNP) selection and allele frequency}

Two SNPs (rs3819024 [promoter], rs2275913 [promoter]) for IL17A gene and four SNPs (rs4819554 [promoter], rs879577 [exon 13, missense Ala367Val], rs2229151 [exon 13, synonymous Lys379Lys], and rs875975 [exon 13, synonymous Ile486Ile]) for IL17RA gene with greater than 0.3 heterozygosity among SNPs located in the promoter or exon (http://www.ncbi.nlm.nih.gov/SNP) were selected. All six selected SNPs were included by the Hardy-Weinberg Equilibrium test (HWE, $p>0.05$ ).

The genotypes were determined by direct sequencing. Genomic DNA was amplified using the following primers: rs3819024 (sense, 5'-GGGTTGGAACATGCCTTTAACA-3'; antisense, 5'-AGCTGCTATGCTATGGGTCAAT-3'; 380 bp); rs2275913 (sense, 5'-TCAAGGTACATGACACCAGAAG-3'; antisense, 5'-GATGAGTTTGTGCCTGCTATGA-3'; 394 bp); rs4819554 (sense, 5'-CACGCGTGCTAAGAAGGAG-3'; antisense, 5'-TATTTTACCCTATACCGGGC-3'; 327 bp); rs879577 (sense, 5'-CCTCAGTTGG GTTTCTCAGC-3'; and antisense, 5'-TCTAGTAGCAGGACACGAGG-3'); rs2229151 (sense, 5'-GGTCAGCATGTGTGGTCTTGT-3'; antisense, 5'-TCAGTACTGGACCCACCCGGC-3'; 327 bp); rs875975 (sense, 5'-CTCTAAGATCATCGTCCTGTG-3'; antisense, 5'-CGAGTACCTGTCCAAGCTCCT- 3'; 327 bp). The samples were sequenced using an ABI Prism 3730XL Analyzer (PE Applied Biosystems, Foster City, CA, USA). Sequence data were analyzed using the SeqManll Software (DNASTAR Inc., Madison, WI, USA).

\section{Statistical analysis}

The HWE for two SNPs was assessed using SNPStats (http://bioinfo.iconcologia.net/index.php) ${ }^{16}$. A linkage disequilibrium (LD) block of polymorphisms was tested using Haploview version $3.32^{17}$. Multiple logistic regression 
models were calculated for the odds ratio, 95\% confidence interval, and corresponding p-values, with controls for gender as a covariable. We used SNPStats, Helixtree Software (Golden Helix Inc., Boezman, MT, USA), and SNPAnalyzer (ISTECH Inc., Goyang, Korea) ${ }^{18}$.

\section{RESULTS}

In this study, 238 AA patients (109 males and 129 females, average age at survey; 28.6 years) were enrolled (Table 1 ). In onset age, one hundred sixty-two patients (68\%) were under age 30 (early onset) and seventy-six patients (32\%) were over age 30 (late onset). Nineteen (8\%) had a family history of AA and 219 (92\%) did not have family history.

Table 1. Demographic and clinical characteristics of AA patients and the control subjects

\begin{tabular}{lcc}
\hline & Alopecia areata & Control \\
\hline Number & 238 & 270 \\
Male/female & $109 / 129$ & $144 / 126$ \\
Age (yr) & $28.6 \pm 13.2$ & $35.7 \pm 11.8$ \\
& $(9 \sim 51)$ & $(11 \sim 48)$ \\
Age of onset & & \\
$\quad<30$ years & 162 & \\
$\quad \geq 30$ years & 76 & \\
Family Hx & & \\
$(+)$ & 19 & \\
$(-)$ & 219 & \\
Type & & \\
Patch & 204 & \\
Totalis or universalis & 34 & \\
Involvement of nail & & \\
$(+)$ & 35 & \\
$(-)$ & 203 \\
Involvement of body hair & & \\
$(+)$ & 35 & \\
$(-)$ & 203 & \\
\hline
\end{tabular}

Values are presented as number or mean \pm standard deviation (range). AA: alopecia areata.
Two hundred and four (86\%) had patchy type of AA and 34 patients (14\%) had alopecia totalis (AT) or alopecia universalis (AU). Thirty-five patients $(15 \%)$ had concomitant nail disorder, but 203 patients $(85 \%)$ did not. Thirty-five patients $(15 \%)$ had an involvement of body hairs such as eyebrow, axillary hairs and pubic hairs, whereas 203 patients $(85 \%)$ did not have body hair involvement.

\section{Association between IL17A/IL17RA gene and AA patients group}

Two SNPs (rs3819024 and rs2275913) in the IL17A gene showed no significant difference between the AA patients group and the control group (Table 2). Also, the LD block in two SNPs of IL17A was not constructed using Gabriel's method. One SNP (rs879577) in IL17RA showed a significant difference between the AA patient group and the control group (Table 2). LD blocks in IL17RA were not constructed using Gabriel's method. A block of strong LD cannot be observed in the region of IL17RA 4 SNPs, which consisted of rs4819554, 879577, 2229151, and 879575.

\section{Age of onset, family history, clinical subtypes, nail involvement and body hair involvement}

We investigated differences in the polymorphisms according to clinical parameters of AA.

\section{Age of onset}

One SNP (rs4819554) of IL17RA gene showed significant difference between the early onset $\mathrm{AA}$ and late onset AA (Table 3). However, two SNPs (rs2275913 and rs3819024) in IL17A did not show significant differences between early onset and late onset AA (Table 3).

\section{Presence of family history}

There were no significant differences of expression for all

Table 2. Logic analysis of IL17A and IL17RA polymorphisms with AA patients and normal control subjects

\begin{tabular}{|c|c|c|c|c|c|c|c|c|c|}
\hline \multirow{2}{*}{$\begin{array}{c}\text { Gene } \\
\text { IL17A }\end{array}$} & \multirow{2}{*}{$\begin{array}{c}\text { SNP (rs) } \\
\text { rs2275913 }\end{array}$} & \multirow{2}{*}{$\begin{array}{c}\text { Region } \\
\text { Promoter }\end{array}$} & \multirow{2}{*}{$\frac{\text { Allele }}{\text { G }}$} & \multicolumn{2}{|c|}{ Case $(\%)$} & \multicolumn{2}{|c|}{ Control (\%) } & \multirow{2}{*}{$\begin{array}{c}p \text {-value } \\
0.5749\end{array}$} & \multirow{2}{*}{$\frac{\text { OR }(95 \% \mathrm{Cl})}{0.9317(0.7265 \sim 1.1940)}$} \\
\hline & & & & 277 & 58.0 & 313 & 57.0 & & \\
\hline & & & A & 197 & 42.0 & 239 & 43.0 & & \\
\hline & rs3819024 & Promoter & A & 268 & 57.0 & 308 & 56.0 & 0.811 & $0.9703(0.7576 \sim 1.2426)$ \\
\hline & & & G & 206 & 43.0 & 244 & 44.0 & & \\
\hline \multirow[t]{8}{*}{ IL17RA } & rs879575 & Exon 13 & G & 466 & 98.0 & 530 & 96.0 & 0.0705 & $0.4727(0.2062 \sim 1.0838)$ \\
\hline & & & A & 8 & 2.0 & 22 & 4.0 & & \\
\hline & rs879577 & Exon13 & G & 454 & 96.0 & 500 & 93.0 & 0.0288 & $0.5237(0.2907 \sim 0.9436)$ \\
\hline & & & A & 20 & 4.0 & 38 & 7.0 & & \\
\hline & rs2229151 & Exon13 & G & 315 & 66.0 & 361 & 65.0 & 0.9617 & $0.9935(0.7627 \sim 1.2943)$ \\
\hline & & & A & 158 & 34.0 & 191 & 35.0 & & \\
\hline & rs4819554 & Promoter & A & 255 & 55.0 & 288 & 53.0 & 0.5353 & $0.9228(0.7158 \sim 1.1897)$ \\
\hline & & & G & 209 & 45.0 & 256 & 47.0 & & \\
\hline
\end{tabular}

IL: interleukin, AA: alopecia areata, SNP: single nucleotide polymorphism, OR: odds ratio, Cl: confidence internal. 
Table 3. Logic analysis of ILI7A and ILI7RA polymorphisms with early onset or late onset AA patients

\begin{tabular}{|c|c|c|c|c|c|c|c|c|c|}
\hline \multirow{2}{*}{$\frac{\text { Gene }}{\text { IL17A }}$} & \multirow{2}{*}{$\begin{array}{c}\text { SNP (rs) } \\
\text { rs2275913 }\end{array}$} & \multirow{2}{*}{$\frac{\text { Region }}{\text { Promoter }}$} & \multirow{2}{*}{$\frac{\text { Allele }}{\text { G }}$} & \multicolumn{2}{|c|}{ Case (\%) } & \multicolumn{2}{|c|}{ Control (\%) } & \multirow{2}{*}{$\frac{p \text {-value }}{0.7645}$} & \multirow{2}{*}{$\begin{array}{c}\text { OR }(95 \% \mathrm{Cl}) \\
1.0620(0.7165 \sim 1.5740)\end{array}$} \\
\hline & & & & 85 & 57.0 & 192 & 60.0 & & \\
\hline & & & A & 65 & 43.0 & 130 & 40.0 & & \\
\hline & rs3819024 & Promoter & A & 81 & 56.0 & 187 & 58.0 & 0.6426 & $0.9112(0.6151 \sim 1.3498)$ \\
\hline & & & G & 69 & 44.0 & 135 & 42.0 & & \\
\hline \multirow{8}{*}{ IL17RA } & rs879575 & Exon13 & G & 146 & 98.0 & 318 & 99.0 & 0.7016 & $0.7306(0.1457 \sim 3.6632)$ \\
\hline & & & A & 4 & 2.0 & 4 & 1.0 & & \\
\hline & rs879577 & Exon 13 & G & 145 & 95.0 & 307 & 96.0 & 0.0641 & $2.2899(0.9319 \sim 5.6268)$ \\
\hline & & & A & 7 & 5.0 & 13 & 4.0 & & \\
\hline & rs2229151 & Exon 13 & G & 95 & 63.0 & 219 & 68.0 & 0.3294 & $0.8125(0.5351 \sim 1.2337)$ \\
\hline & & & A & 55 & 37.0 & 103 & 32.0 & & \\
\hline & rs4819554 & Promoter & A & 81 & 55.0 & 174 & 55.0 & 0.0421 & $1.5074(1.0137 \sim 2.2415)$ \\
\hline & & & G & 67 & 45.0 & 140 & 45.0 & & \\
\hline
\end{tabular}

IL: interleukin, AA: alopecia areata, SNP: single nucleotide polymorphism, OR: odds ratio, Cl: confidence internal.

Table 4. Genotype frequencies of rs879577 in each population

\begin{tabular}{|c|c|c|c|c|c|c|}
\hline \multirow{2}{*}{ Genotype } & \multicolumn{2}{|c|}{ Korean } & \multirow{2}{*}{ European } & \multirow{2}{*}{ Chinese } & \multirow{2}{*}{ Japanese } & \multirow{2}{*}{ Sub-Saharan } \\
\hline & Alopecia areata & Control & & & & \\
\hline GG & 0.92 & 0.86 & 0.383 & 0.822 & 0.800 & 0.183 \\
\hline GA & 0.08 & 0.14 & 0.483 & 0.178 & 0.200 & 0.517 \\
\hline $\mathrm{AA}$ & 0.00 & 0.00 & 0.133 & 0.000 & 0.000 & 0.300 \\
\hline
\end{tabular}

This data is from the SNP database (http://www.ncbi.nlm.nih.gov/projects/SNP). SNP: single nucleotide polymorphism.

SNPs of IL17A and IL17RA gene between the AA patients with family history of $\mathrm{AA}$ and those without (data not shown).

\section{Clinical subtype}

There were no significant differences of expression for all the SNPs in IL17A and IL17RA between patchy type of AA and $A U$ or AT (data not shown).

\section{Nail involvement and body hair involvement}

There were no significant differences of expression for all the SNPs in IL17A and IL17RA between presence of nail involvement and normal nail appearance (data not shown). Also, there were no significant differences in the expression of all SNPs between the presence and absence of body hair involvement (data not shown).

\section{DISCUSSION}

In $\mathrm{AA}$, the origin of the disease process is not fully understood. However, there are indications for a T-cellmediated autoimmune process directed against an unknown autoantigen of the hair follicle. T lymphocytes that have been shown to be oligoclonal and autoreactive are predominantly present in the peribulbous inflammatory infiltrate $^{19}$. It has been hypothesized that AA is an organ- specific autoimmune disease with genetic predisposition and an environmental trigger ${ }^{20,21}$.

Several studies of SNPs in AA have been reported. TaziAhnini et al. ${ }^{22}$ found significant association between the rare allele (C961) of the AIRE polymorphism at position 961 and AA. This association is stronger and more significant with the severe form of the condition (AU). They also examined two polymorphisms in the coding sequence of the Notch4 gene at positions +1297 and +3063 in a case-control study of 116 AA patients and 142 healthy control subjects. The initial analysis showed a significant association of $\mathrm{AA}$ in the overall data set with the Notch4 (T1297C) polymorphism but not with Notch4 $(\mathrm{A}+3063 \mathrm{G})^{23}$. They analyzed polymorphisms of IL-1 ra (IL1RN + 2018) and its homologue IL-1L1 (IL1L1+4734) in a case-control association study on 165 patients and a large number of matched controls. The polymorphisms within IL1RN and IL1L1 themselves or a gene in linkage disequilibrium with IL1RN and IL1L1 predispose to the more severe forms of $\mathrm{AA}^{24}$. The polymorphisms within the MIF-173*C allele confer an increased risk of susceptibility to extensive forms of AA, especially with an early onset of disease. MIF is therefore suggested to be closely implicated in the pathogenesis of more extensive forms of $\mathrm{AA}^{25}$. The present study is, to the best of our knowledge, the first study to investigate a potential influence of IL17A and ILITRA 
gene polymorphisms in AA patients.

The action and expression of IL-17RA on the hair follicle has not been evaluated yet. Therefore, expression of IL-17RA in the hair follicles and its role in pathophysiology of AA should be investigated in the future. The rs879577 SNP which was shown to have significantly different expression in AA patients compared to healthy controls is located in the missense exonal region of the IL17RA gene located on chromosome 22. In this SNP, the AA, AG and GG genotype frequencies are reported respectively to be $0.133,0.483$, and 0.383 in European, $0.000,0.178$, and 0.822 in Chinese, $0.000,0.200,0.800$ in the Japanese population (http://www.ncbi.nlm.nih.gov/SNP; Table 4). We have demonstrated for the first time that ILITRA polymorphisms have the possibilities of determining the pathophysiology of AA or expression of AA phenotypes. In this study, the expression of rs4819554 polymorphism of IL17RA gene can influence the onset time of AA.

\section{REFERENCES}

1. Wiesner-Menzel L, Happle R. Intrabulbar and peribulbar accumulation of dendritic OKT 6-positive cells in alopecia areata. Arch Dermatol Res 1984;276:333-334.

2. McElwee KJ, Yu M, Park SW, Ross EK, Finner A, Shapiro J. What can we learn from animal models of Alopecia areata? Dermatology 2005;211:47-53.

3. Gilhar A, Kalish RS. Alopecia areata: a tissue specific autoimmune disease of the hair follicle. Autoimmun Rev 2006;5:64-69.

4. Shapiro J. Topical immunotherapy in the treatment of chronic severe alopecia areata. Dermatol Clin 1993;11:611-617.

5. Yoshino T, Asada H, Ando Y, Fujii H, Yamaguchi Y, Yoshikawa $\mathrm{K}$, et al. Impaired responses of peripheral blood mononuclear cells to T-cell stimulants in alopecia areata patients with a poor response to topical immunotherapy. $\mathrm{Br}$ J Dermatol 2001;145:415-421.

6. Alkhalifah A, Alsantali A, Wang E, McElwee KJ, Shapiro J. Alopecia areata update: part I. Clinical picture, histopathology, and pathogenesis. J Am Acad Dermatol 2010;62:177-188.

7. Kalish RS, Gilhar A. Alopecia areata: autoimmunity-the evidence is compelling. J Investig Dermatol Symp Proc 2003;8:164-167.

8. Yu JJ, Gaffen SL. Interleukin-17: a novel inflammatory cytokine that bridges innate and adaptive immunity. Front Biosci 2008; 13:170-177.

9. Ghilardi N, Ouyang W. Targeting the development and effector functions of TH17 cells. Semin Immunol 2007;19: 383-393.

10. Aggarwal S, Ghilardi N, Xie MH, de Sauvage FJ, Gurney AL. Interleukin-23 promotes a distinct CD4 T cell activation state characterized by the production of interleukin-17. J Biol Chem 2003;278:1910-1914.

11. Langrish $\mathrm{CL}$, Chen Y, Blumenschein WM, Mattson J, Basham
B, Sedgwick JD, et al. IL-23 drives a pathogenic T cell population that induces autoimmune inflammation. J Exp Med 2005;201:233-240.

12. Harrington LE, Hatton RD, Mangan PR, Turner H, Murphy $\mathrm{TL}$, Murphy $\mathrm{KM}$, et al. Interleukin 17-producing CD4+ effector $\mathrm{T}$ cells develop via a lineage distinct from the $\mathrm{T}$ helper type 1 and 2 lineages. Nat Immunol 2005;6:11231132.

13. Bettelli E, Carrier Y, Gao W, Korn T, Strom TB, Oukka M, et al. Reciprocal developmental pathways for the generation of pathogenic effector $\mathrm{TH} 17$ and regulatory $\mathrm{T}$ cells. Nature 2006;441:235-238.

14. Veldhoen M, Hocking RJ, Atkins CJ, Locksley RM, Stockinger B. TGFbeta in the context of an inflammatory cytokine milieu supports de novo differentiation of IL-17-producing T cells. Immunity 2006;24:179-189.

15. Yao Z, Fanslow WC, Seldin MF, Rousseau AM, Painter SL, Comeau MR, et al. Herpesvirus Saimiri encodes a new cytokine, IL-17, which binds to a novel cytokine receptor. Immunity 1995;3:811-821.

16. Solé X, Guinó E, Valls J, Iniesta R, Moreno V. SNPStats: a web tool for the analysis of association studies. Bioinformatics 2006;22:1928-1929.

17. Barrett JC, Fry B, Maller J, Daly MJ. Haploview: analysis and visualization of $L D$ and haplotype maps. Bioinformatics 2005;21:263-265.

18. Jung MY, Kim BS, Kim YJ, Koh IS, Chung JH. Assessment of relationship between Fyn-related kinase gene polymorphisms and overweight/obesity in Korean population. Korean J Physiol Pharmacol 2008;12:83-87.

19. Hordinsky M, Ericson M. Autoimmunity: alopecia areata. J Investig Dermatol Symp Proc 2004;9:73-78.

20. McMichael AJ. The genetic epidemiol autoimmune pathogenesis alopecia areata. J Eur Acad Dermatol Venereol 1997; 9:36-43.

21. McDonagh AJ, Tazi-Ahnini R. Epidemiology and genetics of alopecia areata. Clin Exp Dermatol 2002;27:405-409.

22. Tazi-Ahnini R, Cork MJ, Gawkrodger DJ, Birch MP, Wengraf $\mathrm{D}, \mathrm{McDonagh} \mathrm{AJ}$, et al. Role of the autoimmune regulator (AIRE) gene in alopecia areata: strong association of a potentially functional AIRE polymorphism with alopecia universalis. Tissue Antigens 2002;60:489-495.

23. Tazi-Ahnini R, Cork MJ, Wengraf D, Wilson AG, Gawkrodger DJ, Birch MP, et al. Notch4, a non-HLA gene in the $\mathrm{MHC}$ is strongly associated with the most severe form of alopecia areata. Hum Genet 2003;112:400-403.

24. Tazi-Ahnini R, Cox A, McDonagh AJ, Nicklin MJ, di Giovine FS, Timms JM, et al. Genetic analysis of the interleukin-1 receptor antagonist and its homologue IL-1L1 in alopecia areata: strong severity association and possible gene interaction. Eur J Immunogenet 2002;29:25-30.

25. Shimizu T, Hizawa N, Honda A, Zhao Y, Abe R, Watanabe $\mathrm{H}$, et al. Promoter region polymorphism of macrophage migration inhibitory factor is strong risk factor for young onset of extensive alopecia areata. Genes Immun 2005;6: 285-289. 